\title{
HIV screening: helping clinicians make sense of test results to patients
}

\author{
Natural frequencies foster insight and should become part of the training of every medical student \\ and HIV counsellor
}

\section{Gerd Gigerenzer director}

Harding Centre for Risk Literacy and Centre for Adaptive Behaviour and Cognition, Max Planck Institute for Human Development, Berlin

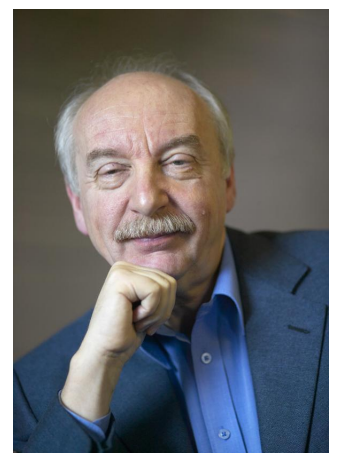

In April 2013 the US Preventive Services Task Force recommended that clinicians screen for HIV infection in people aged 15-65 years, revising its earlier position to screen only people at increased risk and pregnant women. ${ }^{1}$ The proposal elicited discussion about the benefits and harms of antiretroviral treatment, the ethics of testing without people's explicit consent, and much else, but it neglected one crucial issue: risk literacy among clinicians.

When my colleagues and I tested 20 professional HIV counsellors, 10 wrongly asserted that false positive test results never occurred, and eight confused the test's sensitivity (the proportion of people with HIV who actually test positive for it) with its positive predictive value (the proportion of people who test positive who actually have HIV), with only two understanding what a positive test result meant. ${ }^{2}$ In a replication of this study in progress we see little improvement.

Does innumeracy among clinicians matter? No systematic studies of effects on patients exist-just anecdotal reports of people with false positive test results engaging in unprotected sex with other HIV positive people, believing that it would not matter any more, and of people who committed suicide or who endured harmful effects of unnecessary antiretroviral treatment. ${ }^{3}$ A US woman, newly married and pregnant, was told by her doctor to undergo HIV screening and tested positive on western blotting. The doctors told her that the false positive rate was five in 100000 , gave her handouts from the internet about living with HIV, and sent her off to tell her husband and family the news. After a bad evening, she considered her low risk lifestyle and went with her husband to a different clinic for a pinprick test; both partners have tested negative ever since. ${ }^{4}$

How can we help clinicians understand the risk of false positives? Consider a low prevalence group in which the frequency of (undiagnosed) HIV infection is about one in 10 000 , as in female US blood donors. ${ }^{5}$ If the test (such as enzyme immunoassay together with western blotting) has a sensitivity of $99.95 \%$ and a specificity of $99.99 \%$, what is the positive predictive value or $\mathrm{P}$ (HIVlpos)? To calculate this, medical students are taught to insert the prevalence, the sensitivity, and the false positive rate into Bayes's rule:

$\mathrm{P}(\mathrm{HIVlpos})=\mathrm{P}(\mathrm{HIV}) \times \mathrm{P}($ poslHIV $) /[\mathrm{P}(\mathrm{HIV}) \times \mathrm{P}($ pos $\mid \mathrm{HIV})+\mathrm{P}($ no $\mathrm{HIV}) \times \mathrm{P}($ posino HIV $)]$. In our case this gives

$\mathrm{P}($ HIVlpos $)=0.0001 \times 0.9995 /[0.0001 \times 0.9995+0.9999 \times 0.0001]$ or about $50 \%$.

But this formula is not intuitive, which explains why even those who like to point out others' "probability blindness" are sometimes confused themselves, as exemplified by an MIT researcher who wrote that the sensitivity of the HIV test was $87 \%$ and that the false positive rate was "the complement of 87 percent, or 13 percent." A method that has been shown to improve insight is called natural frequencies. ${ }^{7-9}$ These can be represented as a tree (figure). The top of the tree specifies a number of people, say 10000 . In the centre of the tree these are split into one person who is expected to be infected (representing the prevalence) and those who are not. At the bottom these are split again into those who are expected to test positive or negative. Now it is easier to see that among those who test positive one is infected with HIV and one is not (panel A of figure). Thus the positive predictive value is $50 \%$. 


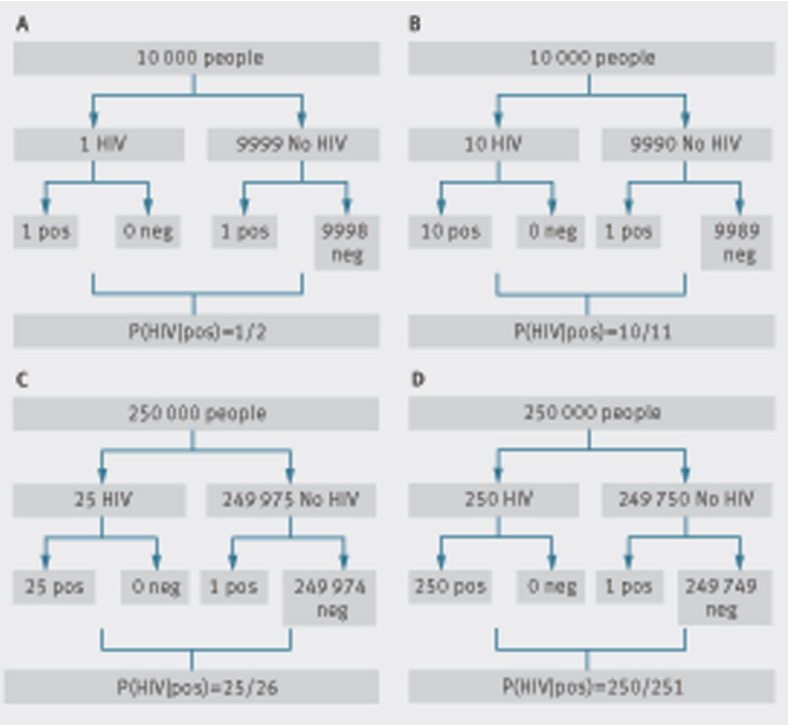

Natural frequencies help to understand the positive predictive value. In panel $A$ the prevalence of HIV is one case in 10000 people screened, and the false alarm rate is one in 10000 . The tree shows that a total of two positive test results is expected: one true positive and one false positive (the positive predictive value is $50 \%$ ). Panel $\mathrm{B}$ shows the same tree for a prevalence of one in 1000 people, resulting in a positive predictive value of $10 / 11$ or $91 \%$. Panels $C$ and D show the same analysis for a false alarm rate of one in 250000

Prevalence, false positive rates, and sensitivity can vary widely in HIV testing, depending on the risk group and the test used. The positive predictive value largely depends on the prevalence of HIV in the screening population and the false positive rate of the test. The figure ignores sensitivity because it is of little use when the incidence of infection is so low. Panel B shows what happens when the prevalence of HIV among those screened increases to one in 1000 (with the same false positive rate).
Here, the positive predictive value increases to $10 / 11$ - that is, of every 11 people who test positive, we expect one false positive. Even if the prevalence is not exactly known, natural frequencies can help us to acquire a feeling for their order of magnitude.

Panels C and D show the same analysis for a test with an extremely low false alarm rate, one in $250000 .{ }^{10}$ Here we need to start with a larger group. In general, the minimum group size at the top of the tree can be determined from the prevalence or the false positive rate, whichever is smaller, and its denominator is then the number on top of the tree. If the prevalence is one in 10000 (panel C), 26 people are expected to test positive, one of them falsely. If the prevalence is one in 1000 (panel D), the positive predictive value increases to $250 / 251$.

Natural frequencies foster insight and should become part of the training of every medical student and HIV counsellor.

Competing interests: None declared.

Provenance and peer review: Commissioned; not peer reviewed.

1 Moyer VA. Screening for HIV: US Preventive Services Task Force recommendation statement. Ann Intern Med 2013;159:51-60.

Gigerenzer G, Hoffrage U, Ebert A. 1998. AIDS counseling for low-risk clients. AIDS Care 1998;10:197-211.

Gigerenzer G. Reckoning with risk. Penguin, 2002.

4 Gigerenzer G. Risk savvy. Penguin [forthcoming].

5 Centers for Disease Control and Prevention. HIV prevalence among selected populations: low-risk populations. CDC. 25 May 2007. www.cdc.gov/hiv/topics/testing/resources/reports/ hiv prevalence/low-risk.htm.

6 Piattelli-Palmarini M. Probability blindness. Bostonia 1991 Mar/Apr:35.

7 Gigerenzer G, Hoffrage U. How to improve Bayesian reasoning without instruction: frequency formats. Psychol Rev 1995;102:684-704.

8 Gigerenzer G, Hoffrage U. Overcoming difficulties in Bayesian reasoning: a reply to Lewis \& Keren and Mellers \& McGraw. Psychol Rev 1999;106:425-30.

9 Akl, EA, Oxman AD, Herrin J, Vist GE, Terrenato J, Sperati F, et al. Using alternative statistical formats for presenting risks and risk reductions. Cochrane Database Syst Rev 2011;3:CD006776

10 Kleinman S, Busch MP, Hall L, Thomson R, Glynn S, Gallahan D, et al. False-positive HIV-1 test results in a low-risk setting of voluntary bold donation: retrovirus epidemiology donor study. JAMA 1998;280:1080-5. 